\title{
Canonical Forms for BiHamiltonian Systems
}

\author{
Peter J. Olver \\ Dedicated to the Memory of Jean-Louis Verdier
}

BiHamiltonian systems were first defined in the fundamental paper of Magri, [5], which deduced the integrability of many soliton equations from the fact that they could be written in Hamiltonian form in two distinct ways. More recently, the classical completely integrable Hamiltonian systems of ordinary differential equations, such as the Toda lattice and rigid body, have been shown to be biHamiltonian systems. (However, recent results of Brouzet, $[\mathbf{1}]$, and extended by Fernandes, [3], indicate that there are global, topological obstructions to the existence of a biHamiltonian structure for a general completely integrable Hamiltonian system.) The connection between biHamiltonian structures and $R$-matrices, [10], which provide solutions to the classical Yang-Baxter equation, has given additional impetus to their study.

Magri's Theorem demonstrates the existence of an infinite hierarchy of commuting Hamiltonians and flows, provided that the two Hamiltonian structures are compatible, in a sense to be defined below. Therefore, any biHamiltonian system of ordinary differential equations will be completely integrable, as long as a sufficient number of the integrals are functionally independent. Explicit, a priori conditions guaranteeing the independence of the integrals are not so evident, and one method of elucidating such conditions is to determine the possible canonical forms for biHamiltonian systems. In [11], Turiel gave a complete classification of "generic" compatible non-degenerate biHamiltonian structures - a "double Darboux Theorem". In [8], this classification was used to find the associated canonical forms for such biHamiltonian systems, and their complete integrability, or lack thereof. The integrability depends on the algebraic structure of the biHamiltonian structure. The main result is that any biHamiltonian system associated with a nondegenerate biHamiltonian structure, each of whose eigenvalues appear in just one irreducible substructure, is necessarily completely integrable; in all other cases, there do exist "non-integrable" biHamiltonian systems. (See below for the precise terminology.)

In this brief survey, I will review these results on the canonical forms for compatible, nondegenerate complex-analytic biHamiltonian systems. Details of these results, as well as some preliminary extensions to the classification of incompatible biHamiltonian structures, can be found in the author's paper [8]. The main outstanding problem in this area is to determine similar canonical forms for degenerate (and compatible) biHamiltonian systems. Unfortunately, Turiel's approach, which is fundamentally tied to the 
covariant differential form framework for symplectic structures, does not appear to readily generalize, since degenerate Poisson structures can only be readily expressed in the contravariant language of bi-vector fields, [7].

A system of differential equations is called biHamiltonian if it can be written in Hamiltonian form in two distinct ways:

$$
\frac{d x}{d t}=J_{1} \nabla H_{1}=J_{2} \nabla H_{0}
$$

Here $J_{1}(x), J_{2}(x)$ are Hamiltonian operators, not constant multiples of each other, determining Poisson brackets: $\{F, G\}_{\nu}=\nabla F^{T} J_{\nu}(x) \nabla G$. The biHamiltonian structure detemined by $J_{1}, J_{2}$ is compatible if the sum $J_{1}+J_{2}$ is also Hamiltonian. The biHamiltonian structure is nondegenerate if the first Hamiltonian operator $J_{1}$ is nonsingular.

Theorem. Suppose $J_{1}, J_{2}$ determine a nondegenerate, compatible biHamiltonian structure. For any associated biHamiltonian system (1), there exists a hierarchy of Hamiltonian functions $\mathrm{H}_{0}, \mathrm{H}_{1}, \mathrm{H}_{2}, \ldots$, all in involution with respect to either Poisson bracket, $\left\{H_{j}, H_{k}\right\}_{\nu}=0$, and generating mutually commuting biHamiltonian flows

$$
\frac{d x}{d t}=J_{1} \nabla H_{k}=J_{2} \nabla H_{k-1}
$$

We classify biHamiltonian structures pointwise according to the algebraic invariants of the skew-symmetric matrix pencil $\lambda J_{1}(x)+\mu J_{2}(x)$ at each $x$. According to the Weierstrass theory, $c f$. [2], the complete system of algebraic invariants of a non-degenerate matrix pencil consists of the eigenvalues, the elementary divisors, and the Segre characteristic. (Degenerate pairs of skew-symmetric matrices are handled by the more detailed Kronecker theory.) A pencil is called elementary if it has just one complex eigenvalue, and irreducible if it has Segre characteristic $[(n n)]$, analogous to a single Jordan block. Every non-degenerate complex matrix pencil can, algebraically, be decomposed into a direct sum of irreducible matrix pencils. (For simplicity, we restrict our attention to complex-analytic systems, although the real case offers little additional difficulty.) The algebraic invariants of a biHamiltonian structure are invariant under the flow of any associated biHamiltonian system. A biHamiltonian structure is generic on a domain $M$ if it has constant Segre characteristic, and the number of functionally independent eigenvalues does not change on $M$.

Theorem. Every generic non-degenerate, compatible biHamiltonian structure can be locally expressed as a Cartesian product of elementary biHamiltonian structures. Every associated biHamiltonian system decomposes into independent subsystems corresponding to the elementary substructures, each of which consists of an autonomous Hamiltonian system 
whose dimension is twice the number of irreducible sub-structures for the given eigenvalue, coupled with a sequence of linear, non-autonomous Hamiltonian systems. In particular, the biHamiltonian system is completely integrable if and only if there is just one irreducible sub-structure for each eigenvalue.

When an eigenvalue is constant, the elementary sub-structure decomposes into a Cartesian product of irreducible sub-structures; however, this decomposition does not hold in the case of non-constant eigenvalues. We will now present the details of the Turiel classification and the structure of associated biHamiltonian systems.

Without loss of generality, we may assume that neither 0 nor $\infty$ is an eigenvalue, so that the biHamiltonian structure is determined by two compatible symplectic Hamiltonian operators. (Otherwise, replace $J_{1}, J_{2}$ by two other linearly independent members of the corresponding pencil.) Darboux' theorem, [7; Theorem 6.22], implies that we can write the first Hamiltonian operator in canonical form

$$
J_{1}=\left(\begin{array}{cc}
0 & I \\
-I & 0
\end{array}\right),
$$

relative to canonically conjugate coordinates $x=(p, q)$. Therefore, only the canonical form of the second Hamiltonian operator needs to be explicitly indicated.

Given a Hamiltonian pair $J_{1}, J_{2}$, any associated biHamiltonian system must be a solution to the linear system of partial differential equations

$$
\nabla H_{1}=M \nabla H_{0}, \quad M=J_{1}^{-1} \cdot J_{2},
$$

where $M$ is the transpose of the recursion operator, [7]. We remark here that the simple system of differential equations (4), which arises in a surprising number of different contexts, is not well understood, except when the matrix $M$ is constant, in which case the general solution can be found in [4]. In the present case, the solutions all have a similar pattern. On any convex open subdomain, the two Hamiltonians $H_{0}, H_{1}$ are expressed as a sum of "basic" Hamiltonians $H_{0}^{(k)}, H_{1}^{(k)}$, which are individually solutions to $(4)$ :

$$
H_{i}(x)=H_{i}^{(0)}(x)+H_{i}^{(1)}(x)+\ldots+H_{i}^{(n)}(x), \quad i=0,1 .
$$

Moreover, each basic pair $H_{0}^{(k)}, H_{1}^{(k)}$, can be most simply expressed in terms of the derivatives with respect to a parameter $s$ evaluated at $s=0$ of a single arbitrary analytic function $F\left(\xi_{1}(x, s), \ldots, \xi_{m}(x, s)\right)$ depending on certain parameterized variables $\xi_{j}(x, s)$. We can therefore summarize the general classification results in this convenient form.

\section{I) Irreducible, Constant Eigenvalue Pairs.}


Canonical coordinates:

$$
(p, q)=\left(p_{0}, p_{1}, \ldots, p_{n}, q_{0}, q_{1}, \ldots, q_{n}\right), \quad n \geq 0
$$

Second Hamiltonian operator:

$$
J_{2}=\left(\begin{array}{cc}
0 & \lambda \mathrm{I}+U \\
-\lambda \mathrm{I}-U^{T} & 0
\end{array}\right)
$$

Here $\lambda \mathrm{I}+U$ denotes an irreducible $(n+1) \times(n+1)$ Jordan block matrix with eigenvalue $\lambda$.

Parametrized variables:

$$
\pi(s)=p_{0}+s p_{1}+s^{2} p_{2}+\cdots+s^{n} p_{n}, \quad \varpi(s)=q_{n}+s q_{n-1}+s^{2} q_{n-2}+\cdots+s^{n} q_{0} .
$$

Basic Hamiltonians:

$$
\begin{array}{ll}
\left.H_{0}^{(k)}(x)=\left.\frac{1}{\lambda} \frac{\partial^{k}}{\partial s^{k}} F_{k}(\pi(s), \varpi(s))\right|_{s=0}+k \frac{\partial^{k-1}}{\partial s^{k-1}} F_{k}(\pi(s), \varpi(s))\right)\left.\right|_{s=0} \\
H_{1}^{(k)}(x)=\left.\frac{1}{\lambda} \frac{\partial^{k}}{\partial s^{k}} F_{k}(\pi(s), \varpi(s))\right|_{s=0}, & 0 \leq k \leq n .
\end{array}
$$

The Hamiltonians are polynomials in the "minor variables" $p_{1}, \ldots, p_{n}$, $q_{0}, \ldots, q_{n-1}$, whose coefficients are certain derivatives of the arbitrary smooth functions $F_{k}\left(p_{0}, q_{n}\right)$ of the remaining two "major variables" $p_{0}$, $q_{n}$. This implies, $c f$. [8], that any biHamiltonian system corresponding to an irreducible, constant eigenvalue biHamiltonian structure is completely integrable, since it can be reduced to a single two-dimensional (planar) autonomous Hamiltonian system for the major variables, with Hamiltonian $n ! F_{n}\left(p_{0}, q_{n}\right)$. (Curiously, the major variables are not canonically conjugate for any of the Hamiltonian structures in the pencil determined by $J_{1}$ and $J_{2}$.) The time evolution of the minor variables is then determined by successively solving a sequence of forced planar, linear Hamiltonian systems in the variables $p_{k}, q_{n-k}$.

\section{Elementary, Constant Eigenvalue Pairs.}

Canonical coordinates:

$$
\begin{gathered}
(p, q)=\left(p^{1}, \ldots, p^{m}, q^{1}, \ldots, q^{m}\right) \\
p^{i}=\left(p_{0}^{i}, \ldots, p_{n_{i}}^{i}\right), \quad q^{i}=\left(q_{0}^{i}, \ldots, q_{n_{i}}^{i}\right),
\end{gathered}
$$

where $n_{1} \geq n_{2} \geq \cdots \geq n_{k} \geq 0$. 
Second Hamiltonian operator:

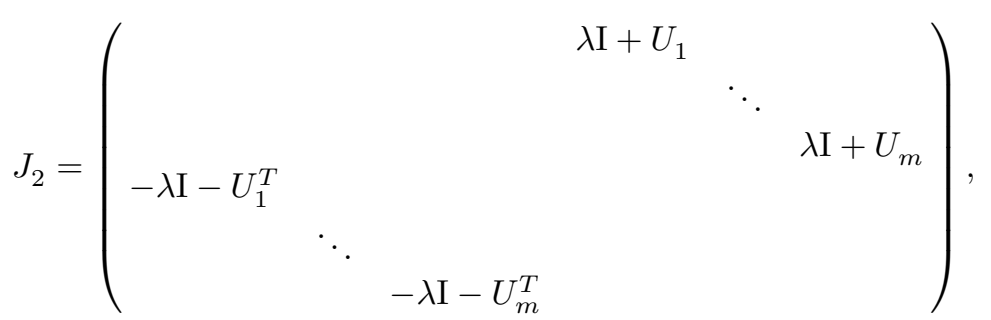

where $\lambda \mathrm{I}+U_{i}$ denotes an irreducible $\left(n_{i}+1\right) \times\left(n_{i}+1\right)$ Jordan block as above.

Parametrized variables:

$$
\begin{aligned}
\pi^{i}(s) & =p_{0}^{i}+s p_{1}^{i}+s^{2} p_{2}^{i}+\cdots+s^{n_{i}} p_{n_{i}}^{i}, \\
\varpi^{i}(s) & =q_{n_{i}}^{i}+s q_{n_{i}-1}^{i}+s^{2} q_{n_{i}-2}^{i}+\cdots+s^{n_{i}} q_{0}^{i} .
\end{aligned}
$$

We define

$$
\pi^{(k)}(s)=\left(\pi^{1}(s), \ldots, \pi^{m_{k}}(s)\right), \quad \varpi^{(k)}(s)=\left(\varpi^{1}(s), \ldots, \varpi^{m_{k}}(s)\right),
$$

where $m_{k}$ denotes the number of indices $n_{i}$ with $n_{i} \geq k$, i.e., the number of irreducible sub-structures of dimension $\geq 2 k+2$; in particular $m_{0}=m$.

Basic Hamiltonians:

$$
\begin{aligned}
& H_{0}^{(k)}(x)=\left.\frac{1}{\lambda} \frac{\partial^{k}}{\partial s^{k}} F_{k}\left(\pi^{(k)}(s), \varpi^{(k)}(s)\right)\right|_{s=0}+ \\
& \quad+\left.k \frac{\partial^{k-1}}{\partial s^{k-1}} F_{k}\left(\pi^{(k)}(s), \varpi^{(k)}(s)\right)\right|_{s=0}, \quad 0 \leq k \leq n_{1} . \\
& H_{1}^{(k)}(x)=\left.\frac{1}{\lambda} \frac{\partial^{k}}{\partial s^{k}} F_{k}\left(\pi^{(k)}(s), \varpi^{(k)}(s)\right)\right|_{s=0}
\end{aligned}
$$

As in the irreducible case, the Hamiltonians are polynomials in the minor variables $p_{j}^{i}, q_{n_{i}-j}^{i}, j \geq 1$, whose coefficients are certain derivatives of arbitrary functions of the major variables $p_{0}^{i}, q_{n}^{i}$. Thus, such a biHamiltonian system reduces to an autonomous $(2 m)$-dimensional Hamiltonian system in the major variables, coupled with a sequence of linear nonautonomous Hamiltonian systems in the appropriate minor variables $p_{k}^{i}$, $q_{n_{i}-k}^{i}, n_{i} \geq k \geq 1$.

\section{Irreducible, Non-constant Eigenvalue Pairs.}

Canonical coordinates:

$$
(p, q)=\left(p_{0}, p_{1}, \ldots, p_{n}, q_{0}, q_{1}, \ldots, q_{n}\right), \quad n \geq 0
$$


Second Hamiltonian operator:

$$
J_{2}=\left(\begin{array}{cc}
0 & P(p)^{-1} \\
-P(p)^{-T} & 0
\end{array}\right)
$$

where $P(p)$ denotes the $(n+1) \times(n+1)$ banded upper triangular matrix

$$
P_{n}(p)=P(p)=\left(\begin{array}{cccccc}
p_{0} & p_{1} & p_{2} & & \cdots & p_{n} \\
& p_{0} & p_{1} & p_{2} & & \\
& & p_{0} & p_{1} & & \vdots \\
& & & \ddots & \ddots & \\
& & & & p_{0} & p_{1} \\
& & & & & p_{0}
\end{array}\right) .
$$

Here $p_{0}$ is the eigenvalue. The explicit formula for the Hamiltonian operator $J_{2}$ in terms of $p_{0}, \ldots, p_{n}$ is quite complicated. However, remarkably, the inverse matrix $J_{2}^{-1}$ is also Hamiltonian, and, in fact, isomorphic to the Hamiltonian structure determined by $J_{2}$; see [8] for an explicit change of variables mapping the one Hamiltonian structure to the other.

Parametrized variables:

$$
\pi(s)=p_{0}+s p_{1}+s^{2} p_{2}+\cdots+s^{n} p_{n}, \quad \varpi(s)=q_{n}+s q_{n-1}+s^{2} q_{n-2}+\cdots+s^{n} q_{0} .
$$

Basic Hamiltonians:

$$
\begin{array}{rlrl}
H_{0}^{(-1)} & =\widetilde{h}\left(p_{0}\right), \quad H_{1}^{(-1)}=h\left(p_{0}\right), \quad \text { where } & \widetilde{h}^{\prime}(s)=\operatorname{sh}(s), \\
H_{0}^{(k)}(x) & =\left.\frac{\partial^{k}}{\partial s^{k}}\left\{\pi(s) \pi^{\prime}(s) F_{k}(\pi(s), \varpi(s))\right\}\right|_{s=0}, & 0 \leq k \leq n-1 . \\
H_{1}^{(k)}(x) & =\left.\frac{\partial^{k}}{\partial s^{k}}\left\{\pi^{\prime}(s) F_{k}(\pi(s), \varpi(s))\right\}\right|_{s=0},
\end{array}
$$

Here $\pi^{\prime}(s)$ is the derivative of $\pi$ with respect to $s$.

In this case, the eigenvalue is a constant, hence $p_{0}$ is a first integral. Once its value is fixed, the other minor variable $q_{n}$ is determined by solving a single autonomous ordinary differential equation. The remaining minor variables $p_{1}, \ldots, p_{n}, q_{0}, \ldots, q_{n-1}$ satisfy a sequence of forced, linear planar Hamiltonian systems.

\section{Elementary, Non-constant Eigenvalue Pairs.}

Canonical coordinates:

$$
\begin{aligned}
(p, q) & =\left(p_{0}, p^{1}, \ldots, p^{m}, q_{0}, q^{1}, \ldots, q^{m}\right), \quad m \geq 2, \\
p^{i} & =\left(p_{1}^{i}, \ldots, p_{n_{i}}^{i}\right), \quad q^{i}=\left(q_{1}^{i}, \ldots, q_{n_{i}}^{i}\right), \quad 1 \leq i \leq m,
\end{aligned}
$$


where $n_{1} \geq n_{2} \geq \ldots \geq n_{k} \geq 1$.

Second Hamiltonian operator:

$$
J_{2}=\left(\begin{array}{cc}
0 & \widehat{P}(p)^{-1} \\
-\widehat{P}(p)^{-T} & 0
\end{array}\right)
$$

where

$$
\widehat{P}(p)=\left(\begin{array}{ccccc}
p_{0} & p^{1} & p^{2} & \ldots & p^{m} \\
& P_{n_{1}-1}\left(\hat{p}^{1}\right) & 0 & \ldots & 0 \\
& & \ddots & & \vdots \\
& & & P_{n_{m-1}-1}\left(\hat{p}^{m-1}\right) & 0 \\
& & & &
\end{array}\right.
$$

Here $\hat{p}^{i}=\left(p_{0}, p_{1}^{i}, \ldots, p_{n_{i}-1}^{i}\right)$, and the $P_{n_{i}-1}$ 's are as given in (5). Again $p_{0}$ is the eigenvalue. Note that this particular biHamiltonian structure is pointwise algebraically reducible, but cannot be decoupled using canonical transformations.

Parametrized variables:

$\pi^{i}(s)=p_{0}^{i}+s p_{1}^{i}+\cdots+s^{n_{i}} p_{n_{i}}^{i}, \quad \varpi^{i}(s)=q_{n_{i}}^{i}+s q_{n_{i}-1}^{i}+\cdots+s^{n_{i}-1} q_{1}^{i}, \quad i \geq 1$.

We further define

$$
\mu^{i}(s)=\frac{\tau^{i}(s)}{s}, \quad \sigma^{i}(s)=\varpi\left(\tau^{i}(s)\right)
$$

where $\tau^{i}(s)$ solves the implicit series equation

$$
\pi^{i}\left(\tau^{i}(s)\right)=\pi^{1}(s), \quad i \geq 2
$$

Let

$$
\mu^{(k)}(s)=\left(\mu^{1}(s), \ldots, \mu^{m_{k}}(s), \quad \sigma^{(k)}(s)=\left(\sigma^{1}(s), \ldots, \sigma^{m_{k}}(s)\right),\right.
$$

where $m_{k}$ denotes the number of indices $n_{i}$ with $n_{i} \geq k$.

The Lagrange inversion formula, [6], implies that the latter two parametrized variables have the alternative expansions

$$
\begin{aligned}
& \mu^{i}(s)=\left.\sum_{n=0}^{n_{i}-1} \frac{s^{n}\left(\zeta^{1}(s)\right)^{n+1}}{(n+1) !} \frac{d^{n}}{d t^{n}}\left[\frac{1}{\left(\zeta^{i}(t)\right)^{n+1}}\right]\right|_{t=0} \\
& \sigma^{i}(s)=q_{n_{i}}^{i}+\left.\sum_{n=0}^{n_{i}-1} \frac{s^{n}\left(\zeta^{1}(s)\right)^{n}}{n !} \frac{d^{n}}{d t^{n}}\left[\frac{1}{\left(\zeta^{i}(t)\right)^{n+1}} \frac{d \omega^{i}(t)}{d t}\right]\right|_{t=0}
\end{aligned}
$$


where $\zeta^{i}(s)=\left(\pi^{i}(s)-p_{0}\right) / s$. The expansions (6) can be expressed in terms of the remarkable nonlinear series differential operator

$$
\mathcal{D}=D^{-1}: e^{s D u}: D=1+\sum_{n=1}^{\infty} \frac{s^{n}}{n !} D^{n-1} u^{n} D, \quad D=\frac{d}{d t}, \quad u=u(t)
$$

with $s$ replaced by $s \zeta^{1}(s)$. In $(7)$, the colons denote normal ordering of the non-commuting operators $D$ and $u$, which is analogous to the so-called "Wick ordering" in quantum mechanics. The operator $\mathcal{D}$ has the surprising property that it commutes with any analytic function $\Phi(u)$, i.e., $\mathcal{D} \Phi(u)=$ $\Phi(\mathcal{D} u)$. See $[\mathbf{9}]$ for details and applications of this operator in combinatorics, orthogonal polynomials and new higher order derivative identities.

Basic Hamiltonians:

$$
\begin{aligned}
H_{0}^{(-1)} & =\widetilde{h}\left(p_{0}\right), \quad H_{1}^{(-1)}=h\left(p_{0}\right), \quad \text { where } \quad \widetilde{h}^{\prime}(s)=s h(s), \\
H_{0}^{(k)}(x) & =\left.\frac{\partial^{k}}{\partial s^{k}}\left\{s \zeta^{1}(s) \frac{d \pi^{1}}{d s} F_{k}\left(\pi^{1}(s), \mu^{(k)}(s), \omega^{1}(s), \sigma^{(k)}(s)\right)\right\}\right|_{s=0}, \\
H_{1}^{(k)}(x) & =\left.\frac{\partial^{k}}{\partial s^{k}}\left\{\frac{d \pi^{1}}{d s} F_{k}\left(\pi^{1}(s), \mu^{(k)}(s), \omega^{1}(s), \sigma^{(k)}(s)\right)\right\}\right|_{s=0},
\end{aligned}
$$

where $0 \leq k \leq n_{1}-1$. In general, such biHamiltonian systems reduce to the integration of a $(2 m-2)$-dimensional autonomous Hamiltonian system for the coordinates $p_{1}^{i}, q_{n_{i}}^{i}, i=1, \ldots, m$, followed by a sequence of forced linear Hamiltonian systems. The eigenvalue $p_{0}$ is constant, and the final coordinate $q_{0}$ is determined by quadrature. Actually, the initial Hamiltonian system can be reduced in order to $2 m-3$ since it only involves the homogeneous ratios of momenta $r^{i}=p_{1}^{i} / p_{1}^{1}, i \geq 2$, as can be seen from the second formula (6) for $\mu^{i}$.

\section{References}

[1] Brouzet, R., Systèmes bihamiltoniens et complète intégrabilité en dimension 4, Comptes Rendus Acad. Sci. Paris 311 (1990), 895-898.

[2] Gantmacher, F.R., The Theory of Matrices, vol. 2, Chelsea Publ. Co., New York, 1959.

[3] Fernandes, R., Completely integrable biHamiltonian systems, preprint, University of Minnesota, 1991.

[4] Jodeit, M. and Olver, P.J., On the equation $\nabla f=M \nabla g$, Proc. Roy. Soc. Edinburgh 116 (1990), 341-358.

[5] Magri, F., A simple model of the integrable Hamiltonian equation, $J$. Math. Phys. 19 (1978), 1156-1162.

[6] Melzak, Z.A., Companion to Concrete Mathematics, Wiley-Interscience, New York, 1973. 
[7] Olver, P.J., Applications of Lie Groups to Differential Equations, Graduate Texts in Mathematics, vol. 107, Springer-Verlag, New York, 1986.

[8] Olver, P.J., Canonical forms and integrability of biHamiltonian systems, Phys. Lett. 148A (1990), 177-187.

[9] Olver, P.J., A nonlinear differential operator series which commutes with any function, SIAM J. Math. Anal. 23 (1992), 209-221.

[10] Semenov-Tian-Shanskii, M.A.., What is a classical R-matrix?, Func. Anal. Appl. 17 (1983), 259-272.

[11] Turiel, F.-J., Classification locale d'un couple de formes symplectiques Poisson-compatibles, Comptes Rendus Acad. Sci. Paris 308 (1989), 575-578.

Peter J. Olver, School of Mathematics, University of Minnesota, Minneapolis, Minnesota, U.S.A. 55455

Research supported in Part by NSF Grant DMS 89-01600. 